\title{
Regulation of Ribosomal RNA Production by RNA Polymerase I: Does Elongation Come First?
}

\author{
Benjamin Albert, ${ }^{1,2}$ Jorge Perez-Fernandez, ${ }^{1,2,3}$ \\ Isabelle Léger-Silvestre, ${ }^{1,2}$ and Olivier Gadal ${ }^{1,2}$ \\ ${ }^{1}$ LBME du CNRS, 118 route de Narbonne, 31000 Toulouse, France \\ ${ }^{2}$ Laboratoire de Biologie Moleculaire Eucaryote, Université de Toulouse, 118 route de Narbonne, 31000 Toulouse, France \\ ${ }^{3}$ Universität Regensburg, Biochemie-Zentrum Regensburg (BZR), Lehrstuhl Biochemie III, 93053 Regensburg, Germany \\ Correspondence should be addressed to Olivier Gadal, olivier.gadal@ibcg.biotoul.fr
}

Received 31 August 2011; Accepted 27 September 2011

Academic Editor: Sebastián Chávez

Copyright (C) 2012 Benjamin Albert et al. This is an open access article distributed under the Creative Commons Attribution License, which permits unrestricted use, distribution, and reproduction in any medium, provided the original work is properly cited.

\begin{abstract}
Ribosomal RNA (rRNA) production represents the most active transcription in the cell. Synthesis of the large rRNA precursors (35-47S) can be achieved by up to 150 RNA polymerase I (Pol I) enzymes simultaneously transcribing each rRNA gene. In this paper, we present recent advances made in understanding the regulatory mechanisms that control elongation. Built-in Pol I elongation factors, such as Rpa34/Rpa49 in budding yeast and PAF53/CAST in humans, are instrumental to the extremely high rate of rRNA production per gene. rRNA elongation mechanisms are intrinsically linked to chromatin structure and to the higherorder organization of the rRNA genes (rDNA). Factors such as Hmol in yeast and UBF1 in humans are key players in rDNA chromatin structure in vivo. Finally, elongation factors known to regulate messengers RNA production by RNA polymerase II are also involved in rRNA production and work cooperatively with Rpa49 in vivo.
\end{abstract}

\section{Introduction}

In cell nuclei, three RNA polymerases transcribe the genome. The most importance is placed on RNA polymerase II (Pol II), which is responsible for synthesizing mRNA and a large variety of noncoding RNAs. The vast majority of RNA production in growing cells is carried out by RNA polymerase I (Pol I), which transcribes the precursor of large rRNA, and by RNA polymerase III (Pol III), which transcribes $5 \mathrm{~S}$ rRNA, tRNA, and some noncoding RNAs. Observation of cryofixed cryosubstituted other sections analyzed by electron microscopy reveals that exponentially growing budding yeast cells contain up to $10^{4}$ ribosomes per $\mu \mathrm{m}^{3}$ [1], which represents up to $10 \%$ of the cytoplasmic volume [2] (Figure 1(a)).

An early step in ribosome biogenesis is initiated by the extremely high transcriptional activity of Pol I and occurs in the largest nuclear domain, the nucleolus (Figure 1(b)). Electron microscopy of nuclear/nucleolar chromatin dispersed by Miller spreading allowed rRNA gene transcription and cotranscriptional assembly to be visualized directly at the single gene level [3] (Figure 1(d)). rDNA is organized in head-to-tail tandem arrays of rRNA genes [4] contained in budding yeast between 100 and 200 copies per cell [5], and from 200 to 300 per mammalian haploid genome [6]. Analysis of transcribed ribosomal DNA (rDNA) after Miller spreading revealed that up to 150 Pol I enzymes simultaneously transcribe rRNA genes in mutant with only 25 rRNA genes [1, 7] (Figures 1(c) and 1(d)). Importantly, despite being the most highly transcribed genes of the genome, rDNA is subject to epigenetic regulation, and only some rRNA genes are transcriptionally active [8]. In this paper, we will focus on recent advances made in understanding the regulation of Pol I activity, including elongation in the context of ribosome assembly.

\section{Is Regulation of Pol I Initiation the Only Regulated Step in rRNA Production In Vivo?}

Eukaryotic RNA polymerases are able to recognize promoters only when these sequence elements are associated with 


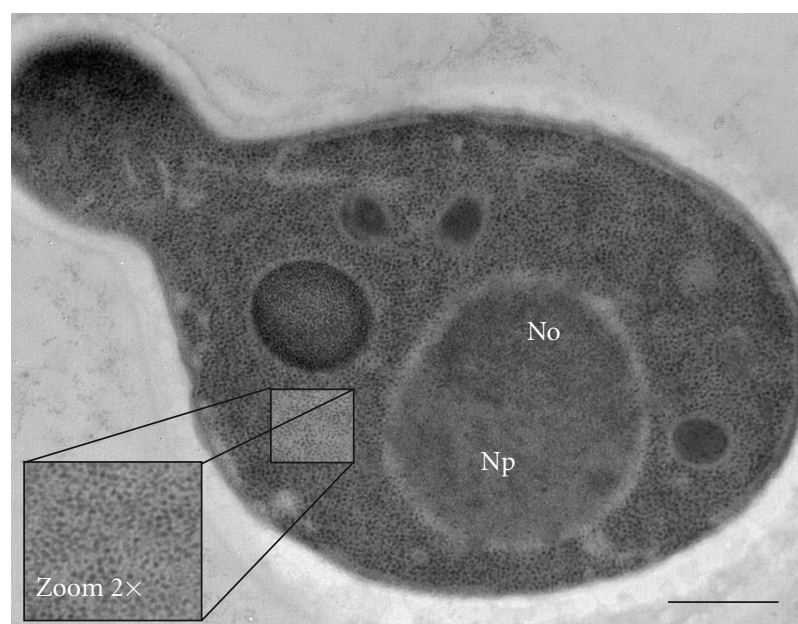

(a)

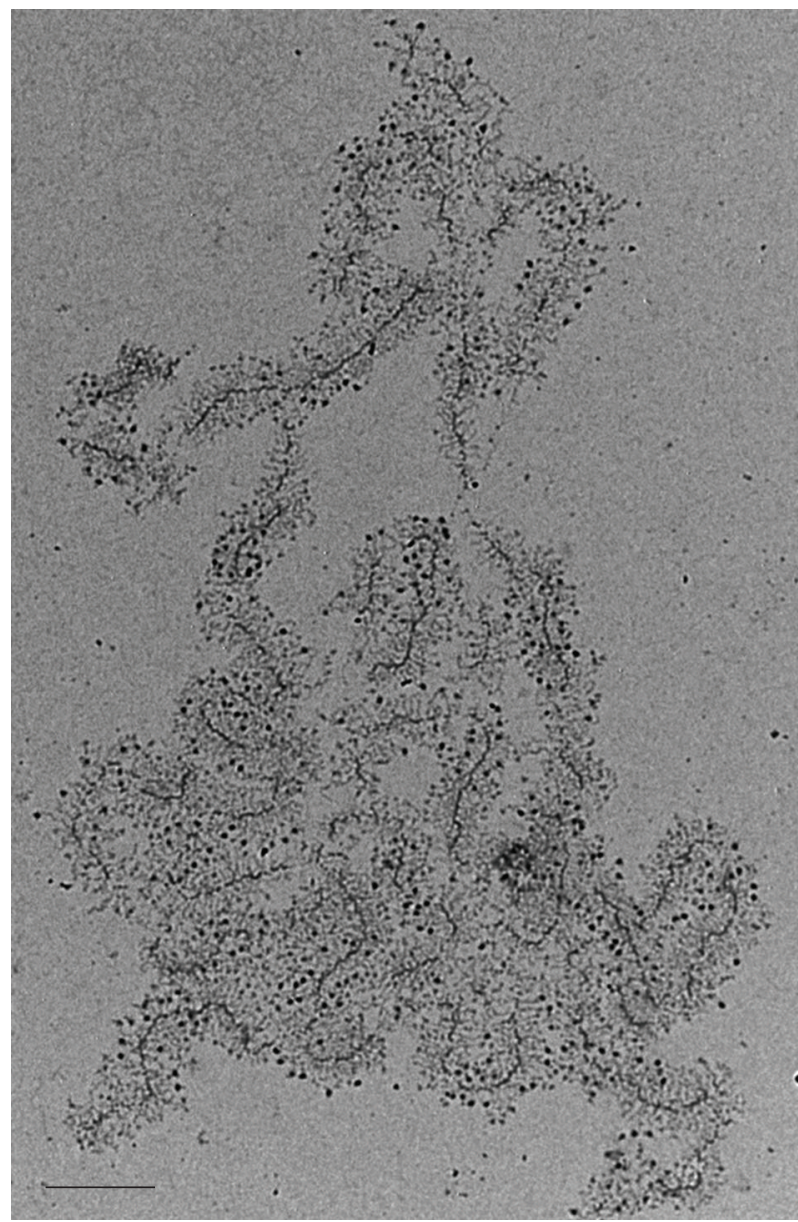

(c)

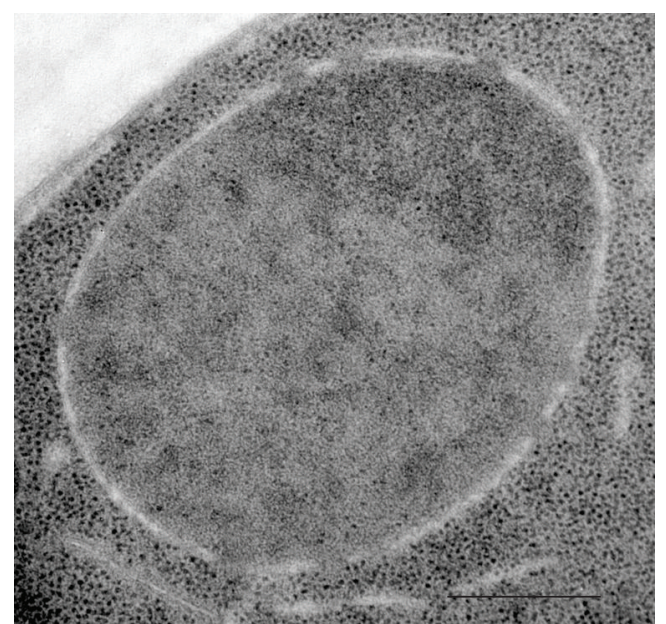

(b)

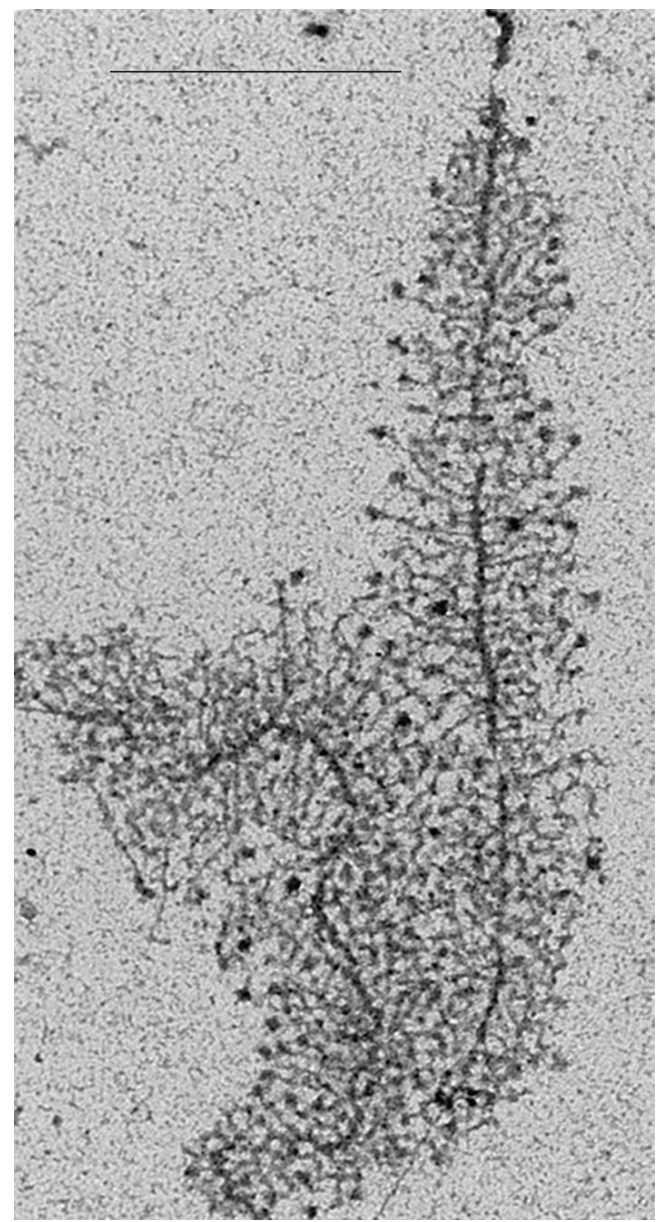

(d)

FIGURE 1: Budding yeast cells and ribosome production. (a) Morphology of Saccharomyces cerevisiae cells after cryofixation and freeze substitution. Ribosomes are individually localized in the cytoplasm (see individual ribosomes detected in the zoomed region). In the nucleus, the nucleolus (No) is detected as a large electron-dense region compared with low electron density of the nucleoplasm (Np). (b) Morphology of the nucleolus. The nucleus appears outlined by a double envelope with pores, and the nucleolus is in close contact with the nuclear envelope. In the nucleolus, a dense fibrillar network is visible throughout the nucleolar volume. Granular components are dispersed throughout the rest of the nucleolus. (c) Visualization of active genes in rDNA. Using a mutant strain with a reduced number of rDNA copies (strain NOY1071; 25 rDNA copies), Miller spreading of total nucleolar DNA allowed single-gene analysis of rRNA genes. (d) Quantification of actively transcribed rDNA. Using high magnification, we can detect individual polymerases associated with nascent rRNA. Bars represent $500 \mathrm{~nm}$. 


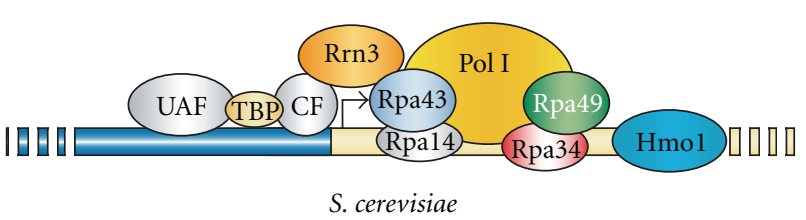

(a)

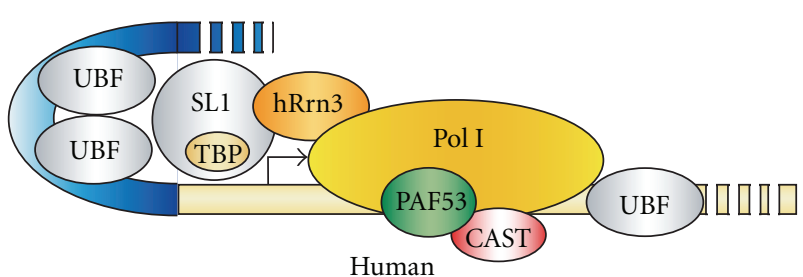

(b)

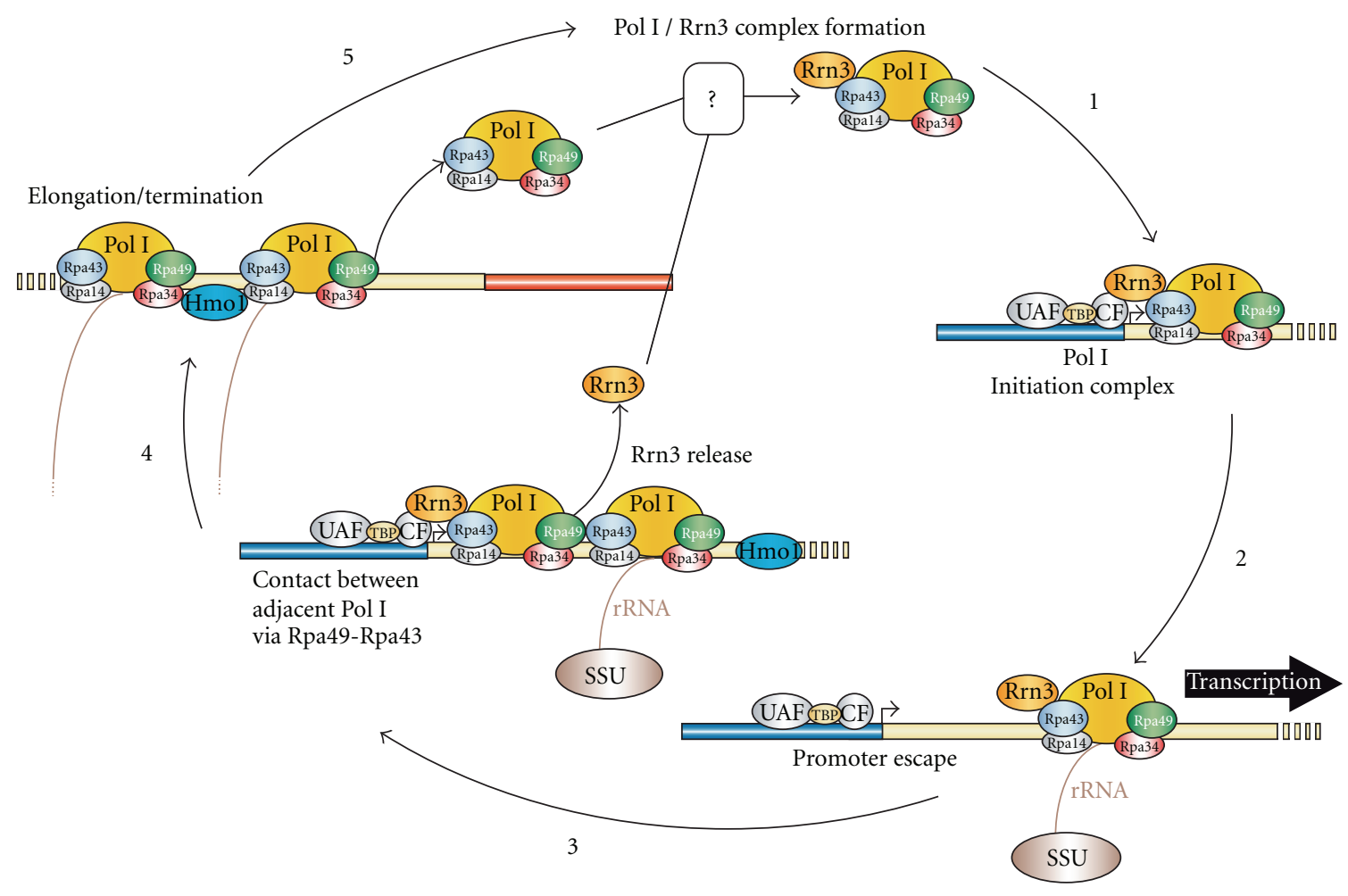

(c)

Figure 2: Schematic representation of the Pol I transcription cycle. Simplified composition of the Pol I preinitiation complex (PIC) in (a) budding yeast and (b) human cells. The Pol I transcription cycle in budding yeast. (1) Recruitment of Rrn3/Pol I onto an rDNA promoter associated with the SL1 and UAF complex allows PIC formation. (2) Promoter escape and rRNA synthesis are coupled with cotranscriptional recruitment of the SSU processome. (3) Rrn3 dissociation is achieved by the formation of an adjacent PIC. Pol I subunits Rpa49 and Rpa43 from the adjacent polymerases promote Rrn3 release from the transcribing Pol I. (4) Pol I transcription of rRNA is coupled with nascent rRNA processing and termination. (5) Pol I holoenzyme is recycled by reassociation with Rrn3, an as yet uncharacterized regulatory process. Hmol function during elongation remains to be clarified, but is revealed by a tight genetic interaction with Pol I elongation mutant rpa49s (4 and 5).

specific initiation factors. Pol I initiation factors have been characterized for both humans and yeast (Figure 2(a)). In mammals, selectivity factor 1 (SL1) in humans and TIF-1B in mice are composed of the TATA-binding protein (TBP) and four TBP-associated factors (TAFs), bound to the core promoter [9-14]. Upstream binding factor (UBF) acts as a dimer and induces a loop formation called the enhanceosome, which brings the activating sequence into close proximity with the core promoter element $[15,16]$. UBF binding stabilizes the association of SL1/TIF-1B with promoter elements [9]. A recent study suggested that UBF bound after SL1 binding and during promoter escape by Pol I [17]. UBF is also bound to the transcribed region [18] and can regulate Pol I elongation [19]. Additionally, UBF and SL1 are regulated by posttranslational modifications. Active Pol I enzymes are associated with numerous other factors such as TFIIH, protein kinase CK2, nuclear actin, nuclear myosin 1 (NM1), chromatin modifiers G9a and SIRT7 and with proteins involved in replication and DNA repair: Ku70/80, proliferating cell nuclear antigen, and CSB. For most factors, mechanistical insights are lacking (see [20] for a recent review).

In budding yeast, a core factor (CF) associates with the Pol I promoter, and this binding is stabilized via TBP by an upstream-associated factor, or UAF [21-27]. CF and SL1 are likely to be functionally equivalent. In contrast, yeast UAF and mammalian UBF both interact with upstream stimulatory elements but have very different functions. UBF1 
also regulates Pol I elongation [19]. The S. cerevisiae HMGBox protein, Hmo1, is associated with the Pol I-transcribed region and is able to rescue growth of the Pol I elongation mutant rpa49 [28]. Therefore, UBF1 and Hmol might have a conserved function in stimulating Pol I elongation (Albert et al. submitted).

Surprisingly, both human and yeast Pol I enzymes are unable to initiate productive RNA synthesis with only promoter-bound factors $[29,30]$. Only a minor fraction of free Pol I is associated with an additional initiation factor: Rrn3 in yeast, hRrn3 in humans, or TIF-1A in mice (Figure 2(b)). When Pol I associates with one of these factors, it recognizes the promoter-bound factors and forms a preinitiation complex (PIC) [30-35].

The amount of Pol I-Rrn3p complexes represents a limiting step in transcription initiation, but how this association is achieved and regulated remains a major research topic [36]. Numerous signaling pathways target Pol I activity in vivo. The target of rapamycin complex 1 (TORC1) regulates ribosome production in response to nutriment availability [37]. Upon inhibition of TORC1 by rapamycin or during stationary phase, the amount of Pol I-Rrn3 complex drops $[31,38,39]$. The regulatory function of Rrn3's association with Pol I was demonstrated by producing an artificial fusion protein of Rrn3 joined to its interacting Pol I subunit, Rpa43. In a partially purified in vitro system, this fusion, called CARA, led to a constitutively active Pol I even during stress, showing that Pol I complexed with Rrn3 is initiation competent even under conditions known to inhibit ribosome production [39]. This initial observation suggested that a deregulated initiation event is sufficient to generate constitutively active Pol I in vivo. However, other findings are now challenging this initial regulatory model based on the availability of an Rrn3-Pol I complex. Rrn3 function is not restricted to initiation only, and it is also involved in a postinitiation step of the Pol I transcription cycle. Rrn3 is released from Pol I during postinitiation, and this process requires Rpa49, another Pol I-specific subunit [40]. In the absence of Rpa49, the CARA mutant is not viable [40]. Therefore, when Rrn3 is physically tethered to Pol I, Rpa49 function becomes essential [40], which suggests the existence of a functional interaction between Rpa49 and Rrn3 after Pol I recruitment [1]. Initial studies suggested that the interaction between Rrn3 and another Pol I-specific subunit, Rpa43, is regulated by phosphorylation [34, 41]. A mutational analysis of Pol I did not reveal the specific residues involved in this regulation but did not exclude the involvement of phosphorylation [42]. Recent works from the Tschochner's Laboratory have demonstrated that Rrn3 is destabilized by a PEST domain, a peptide sequence rich in proline $(\mathrm{P})$, glutamate $(\mathrm{E})$, serine $(\mathrm{S})$, and threonine $(\mathrm{T})$, in its N-terminal domain $[43,44]$. A nondegradable form of Rrn3, missing this PEST motif, attenuated the reduction in initiation competent Pol I-Rrn3p complexes observed upon nutrient depletion. Such a mutation should mimic the CARA mutant phenotype. Unfortunately, this non-degradable form of Rrn3 associated with Pol I has not been tested in vitro in a partially purified extract. Nevertheless, in this background,
rRNA synthesis was downregulated in vivo upon nutrient depletion [44]. Additionally, although levels of the Rrn3-Pol I complex are depleted during stress, the amount remaining is sufficient to observe ongoing initiation events. Therefore, Pol I activity is not regulated only by the initiation competent Rrn3-Pol I complex, but is likely to be influenced by nascent ribosomal assembly. An elegant study suggested that downregulation of ribosomal protein production could also result in a rapid decay of newly made rRNA in vivo [45]. Indeed, Sch9, which acts downstream of TORC1, targets ribosomal protein gene (RPG) transcription as well as rRNA production by Pol I $[46,47]$. Rrn3 might also impact rRNA processing since a mutant of Cbf5, the pseudouridine synthetase that modifies rRNA, is rescued by Rrn3 overexpression [48]. Along the same lines, accumulation of RPG mRNA in the CARA mutant background was resistant to repression by TORC1 inhibition [39]. In fission yeast, a subunit of the Rrn7 core factor also binds RPG promoters, suggesting a coupling between rRNA production and RPG transcription [49]. In budding yeast, Hmol, bona fide Pol I transcription factors, also bind most RPG promoters [50]. Stochiometric production of all ribosomal constituents is tightly controlled and is probably achieved at multiple levels [2]. Is Pol I initiation the only regulated step in rRNA production in vivo? Although the association of Rrn3 with Pol I is a very important regulatory step, it is only one of the numerous pathways that regulate Pol I activity. In this paper, we will extensively describe how the rRNA elongation step might be regulated to integrate all the complex processes necessary to achieve this early step of ribosome assembly.

\section{Early Ribosome Assembly Occurs during rDNA Transcription}

RNA synthesis in the nucleus is invariably coupled with the recruitment of specific proteins shortly after synthesis and leads to the formation of large ribonucleoproteins (RNPs). Strikingly, the fate of the RNA depends on the RNA polymerase synthesizing the transcript. Through their association with the transcribing polymerase appropriate RNA-interacting proteins are driven into the local proximity of the newly synthesized RNA. The COOH-terminal domain (CTD) of the largest subunit of Pol II is the best example of this mechanism [51]. The CTD can recruit the pre-mRNA capping, splicing, and $3^{\prime}$-processing machinery, which are then tethered together with the pre-mRNA. Often, the same factors affecting early RNP assembly, maturation, and export also regulate Pol II elongation, which effectively bridges these processes $[52,53]$. Cotranscriptional assemblies of RNP particles are well known to impact the fate of the transcribed RNA. Even more important, polymerase elongation rates can determine the nature of mature mRNA products, as shown by alternative splicing that depends on the elongation rate [54].

Pol I transcription provided the first example of cotranscriptional RNP particle assembly [3]. Paradoxically, the intimate connection between early RNP assembly and Pol I elongation has been suggested only recently. From Oskar 
Miller's original 1969 description of a transcribed rRNA gene as a Christmas tree, in which the nascent RNA cotranscriptionally assembled with maturation factors that appeared as decorating "terminal balls," 33 years elapsed before the molecular nature of the terminal balls in budding yeast was fully unveiled by the laboratories of Ann Beyer and Susan Baserga [3]. The terminal balls were renamed SSU processomes [55] and are early preribosomal particles, which contain U3 snoRNA and a set of proteins called the UTPs ( $U$ three proteins). An intimate relationship between early assembly and transcription was then suggested from a study of a UTP subgroup, the tUTPs, for transcription-UTP [56]. The tUTPs form a complex with a protein composition very similar to the UTP-A complex [57] with only one distinction: the presence of either Utp 5 or Pol5, respectively. tUTP/UTP-A is recruited to the chromatin independently of transcription; is required for efficient accumulation of Pol I transcripts; has been suggested to be essential for Pol I transcription in a run-on assay [56]. Alternatively, tUTP was suggested to be required for early pre-rRNA stabilization. In the absence of being complexed with tUTP/UTP-A, nascent RNA transcripts are targeted by TRAMP (Trf4/Air2/Mtr4p Polyadenylation) complex and degraded by the nuclear exosome [58]. In human cells, recruitment of human tUTP orthologs to NORs (nucleolus organizer regions) occurs independently of transcription but depends on the protein UBF [59]. Miller spreads have revealed that nascent rRNAs are not only assembled cotranscriptionally with a large set of proteins, but are also cleaved cotranscriptionally in E. coli [60], Dyctiostelium [61], and budding yeast [62]. This cotranscriptional rRNA cleavage has been independently confirmed and quantified using in vivo labeling approaches [63]. Following tUTP/UTP-A recruitment, cotranscriptional assembly is a stepwise and highly hierarchical process [64] that utilizes preexisting autonomous building blocks. These entities sequentially interact with the pre-rRNA and exhibit different interdependencies with respect to each other. This model has been validated and extended by different groups [65].

\section{Pol I Elongation Rate, rRNA Cotranscriptional Maturation, and Topological Stress}

When driven by a strong Pol II promoter, ribosomal DNA can be transcribed by Pol II [66]. Functional ribosomes can ultimately be produced without Pol I, but this is accompanied by a drastic phenotype. Yeast lacking Pol I activity can survive with such an artificial rDNA construct but grow very poorly (i.e., have a doubling time 4 to 5 times longer than normal) and have a massively altered nucleolar and nuclear morphologies $[67,68]$. Despite being not strictly essential, the Pol I elongation rate must be properly controlled for efficient ribosome production.

Biochemical purification of Pol I activity copurified a large fraction of the rRNA maturation machinery [69]. Early maturation factors such as Prp43, Nop56, Nop58, or Gno1 have been shown by two-hybrid assay to be physically tethered to the Pol I-specific subunit Rpa34 [40]. The loading of
tUTP/UTP-A onto nascent transcripts is facilitated by its association with Pol I promoters prior to pre-rRNA [56, 59] and is required either for transcription or for stabilizing nascent transcripts [58]. The first direct evidence that elongation rate regulation is required for proper ribosomal assembly came from a study of an elongation mutant by David Schneider in the laboratory of Masayasu Nomura [70]. In a mutant bearing a single substitution (rpa135-D784G) in the second largest subunit of Pol I, near the active center of the enzyme, rRNA processing was affected with the greatest defect occurring in the 60S assembly pathway [70]. This suggested that the stochiometric production of the $40 \mathrm{~S}$ and $60 \mathrm{~S}$ subunits depends on the elongation rate of Pol I.

The structural analysis comparing the three nuclear RNA polymerases revealed one feature that distinguished Pol I from the other two (Pol II and Pol III): the presence of the heterodimer Rpa49/Rpa34 [71]. Rpa49 and Rpa34 form a heterodimer that is structurally similar to the one formed by two Pol II transcription factors, TFIIE and TFIIF. The hPAF53/CAST heterodimer is the human ortholog of Rpa49/Rpa34 [72]. We recently uncovered how Rpa49 releases Rrn3 from Pol I during elongation, despite the diametric opposition of these proteins in the Pol I complex $[1,71]$. With such a configuration, Rpa49 and Rrn3 are unlikely to interact directly with each other in the Pol I complex. However, the very high loading rate of Pol I per rRNA gene leads to extensive contact between adjacent Pol I enzymes (Figure $1(\mathrm{~d})$ ). Interactions between adjacent Pol I enzymes along the rDNA place Rpa49 and Rpa43 in close proximity, thus allowing Rpa49 to interact with the Rpa43 subunit on a nearby Pol I to promote the release of Rrn3 from Rpa43 [1]. In our model, the Pol I/Rrn3 complex dissociates only after another Pol I starts transcribing rRNA and reflects cooperativity between Pol I enzymes (Figure 2(b)). When we measured distribution of Pol I along rDNA using Miller spreading, we observed a striking degree of enzyme clustering, with $60 \%$ of the enzymes in direct contact, which is compatible with our model. Additionally, this clustering was inhibited by deletion of the Rpa49 gene. What are the consequences of this clustering? By opening the DNA duplex, DNA in front of RNA polymerase becomes overwound, or positively supercoiled; the DNA behind the polymerase becomes underwound, or negatively supercoiled [73]. Importantly, when multiple polymerases are in close proximity, topological distortion of the DNA duplex in front of and behind each adjacent polymerase would be compensated, resulting in topological stress only in front of the first polymerase, and after the last. Therefore, the absence of clustering of adjacent polymerases in the rpa49D mutant should lead to massive rDNA supercoiling. Indeed, Rpa49 deletion has a tight genetic relationship with the two type I yeast topoisomerases: Top 1 and Top3 $[40,74]$. Top3, in complex with Sgs1, is required for the stability of rDNA in vivo and is genetically linked to Rpa49 [74, 75]. Top1 is also involved in rDNA stability [76] and seems to be directly involved in rRNA production. Rpa34 has also been found to interact directly with Top1 in two-hybrid assays [40].

The extremely high transcription rate of Pol I should lead to extensive torsional stress on the rDNA template. 
Is topological stress a selective feature of Pol I transcription? A good answer to this question came from the study using actinomycin $\mathrm{D}$, a DNA-intercalating agent widely used in vivo as a Pol I inhibitor in metazoan cells. The transcription of rRNA genes in mammalian cells is about 50-100-fold more sensitive to actinomycin $\mathrm{D}$ than the synthesis of small RNAs and heterogeneous nuclear RNA [77]. However, Pol Iselective inhibition by actinomycin $\mathrm{D}$ is not inherent to the Pol I transcriptional machinery. Actinomycin D has no effect at low concentrations in vitro or in a transfected reporter system [78]. In fact, low levels of actinomycin D stimulate Pol I rRNA initiation events in vivo; Pol I elongation, however, is strongly inhibited [79]. Such surprising activity can be explained by the ability of low actinomycin $\mathrm{D}$ concentrations to stabilize DNA/topoisomerase interactions [80]. The specific inhibition of rRNA synthesis is at least in part due to the close relationship between topoisomerase and Pol I activities in vivo.

In yeast, a top 1 deletion mutant led to the formation of R-loops (DNA-RNA hybrids) within transcribed rRNA genes [81]. These rRNA production defects, with accumulation of rRNA intermediates ending in G-rich stretches of the $18 \mathrm{~S}$ rRNA, are similar to those observed in the Pol I elongation mutants rpa135-D784G and rpa49D (discussed in [70, 81]). Additionally, it was reported that the maximum transcription rate of rDNA by Pol I in a top 1 deletion mutant generates massive negative supercoiling of rDNA template, which was revealed by the presence of DNA template melting in an A-T-rich region of rDNA that was detectable in Miller spreads [82]. Top2 is essential for growth, but top2 mutants primarily succumb to mitotic failure. Top2 function in rRNA transcription can be studied in Top2 mutants kept in G1 phase and prevented from entering mitosis. Top1 and Top2 cooperate in Pol I transcription [83] but in contrast to a top1 deletion mutant, a top2-ts mutant accumulates positive supercoiling, which inhibits elongation by Pol I [82]. The difference between these Top1 and Top2 phenotypes is surprising since both topoisomerases are able to relax positively and negatively supercoiled DNA. The difference may be related to the intrinsic properties of each enzyme and to the ability of the chromatin to transiently absorb either negative or positive torsional stress [84]. Top1 is a torque-sensitive topoisomerase with a poor ability to relax chromatin. It functions mainly in the relaxation of the negative supercoiling produced in the wake of Pol I $[85,86]$. In contrast, Top2 is likely to be more efficient in relaxing the positive supercoiling produced ahead of Pol I, because no sink for torsional stress, such as melting of the DNA template, exists in this case [84]. Finally, genetic interactions between Top 1 and Trf4/Trf5, two members of the TRAMP complex [87], have been reported previously, and these might be explained by the presence of unresolved R-loops that accumulate in the absence of nuclear rRNA degradation machinery.

\section{Epigenetic Regulation and Chromatin Status of rDNA}

In eukaryotes, rRNA genes are either organized in a closed chromatin state in which they are transcriptionally inactive in transcription or are in an open chromatin state [8]. In mammals, inactive rRNA genes are subject to DNA methylation (for review, see [20]). In budding yeast, which lacks DNA methylation, a proportion of rRNA genes is also transcriptionally repressed.

Active rDNA with an open chromatin structure can be distinguished from inactive rRNA genes by psoralen crosslinking. Psoralen is an intercalating reagent which crosslinks to DNA under UV irradiation. The nucleosomes associated with inactive DNA lock the DNA topology and prevent psoralens from crosslinking to DNA. When rDNA is subjected to psoralen treatment and crosslinking, two types of bands are detected: a slow- and a fast-migrating band $[8$, 88]. The molecular identity of the proteins associated with both types of rDNA molecules was unambiguously established using psoralen combined with ChEC (chromatin endogenous cleavage) $[89,90]$. In ChEC methods, rDNA-associated proteins are expressed as recombinant proteins fused to micrococcal nuclease (MNase). After formaldehyde crosslinking and psoralen treatment, MNase is activated and cleaves either the fast- or slow-migrating rDNA band. The fast-migrating band corresponds to transcriptionally inactive rDNA, which is enriched in nucleosomes [90]. The slow-migrating band is transcriptionally competent $\mathrm{rDNA}$, enriched in Pol I, depleted of histones (compared to genomic DNA), and bound by Hmol, a yeast protein similar to UBF [90]. In budding yeast, the number of rRNA genes in open chromatin seems not to be a major regulatory determinant for Pol I activity [7]. rDNA copy number can change from cell to cell as a result of unequal crossing over and ranges between 100 and 200 repeats in a population [91]. Fob1 is required for this chromosomal instability [92]. In the absence of Fob1, cell populations with a stable number of rDNA repeats could be generated [7]. With 42 copies, or even more so with only 25 copies, most rRNA genes were active and in an open chromatin state. With an rDNA copy number as low as 25 actively transcribed rRNA genes, growth was not affected, but more polymerases were loaded on each active gene [7].

During the cell cycle, the ratio of open to closed rDNA changes. Newly replicated rDNA becomes psoralen inaccessible and shows nucleosome assembly on both strands after the passage of the replication forks [93]. Following replication, the amount of open chromatin was found to steadily increase at all stages of the cell cycle, including during cell cycle arrest [94]. This increase required Pol I activity. The maintenance of the open chromatin state did not require Pol I activity, but $\mathrm{Hmol}$ inhibited replication-independent nucleosome assembly [94].

In sharp contrast to ChEC data, chromatin immunoprecipitation (ChIP) analyses targeting $\mathrm{H} 3$ and $\mathrm{H} 2 \mathrm{~B}$ histones in mutant strains harboring most rRNA genes in an open chromatin state (42 or $25 \mathrm{rDNA}$ copies) strongly suggested that significant quantities of histone molecules are present on active rRNA genes [95] (Albert et al. Submitted).

Both ChIP and psoralen-ChEC have some intrinsic limitations. ChIP is known to be sensitive to background binding, which can lead to false-positive detection. Conversely, ChEC is prone to false-negative detection [89]. After MNase 
activation, MNase fusion proteins are released from genomic DNA after cleavage of the DNA molecule. These released proteins are then able to cleave genomic DNA at nonspecific sites [89]. ChEC experiments must be performed in a carefully controlled time-course. Due to the abundance of histones molecules, cleavage time is kept bellow 15 minutes [90]. Open rRNA genes appear more resistant to MNase digestion than genes in closed rDNA or naked plasmid DNA, but cleavage is detectable [90]. With such experimental limitations, ChEC combined with psoralen can be used to compare relative levels of histone enrichment, but cannot be used to conclude whether histone is present or absent on open chromatin.

Due to such intrinsic technical limitations, the exact composition of rDNA in the open chromatin state is still widely debated $[90,95]$. The analysis of actively transcribed versus untranscribed rDNA can also be performed using Miller spreading (see Figure 1(c)). However, quantifying the ratio of active versus inactive rRNA genes is intrinsically biased, as it underestimates the fraction of inactive rDNA. Transcriptionally inactive rRNA genes are not directly detectable, but can be indirectly visualized because they are flanked by active rRNA genes. This method allows rRNA genes to be characterized at the single-gene level. From such analyses, two important conclusions were reached: nucleosomes are not detectable on actively transcribed genes (data not shown) and nucleosome structures are detectable on some inactive rDNA genes but not all (Figure 3).

The presence of histones on open chromatin, as detected by ChIP, contrasts sharply with the absence or strong depletion of nucleosomes on open chromatin that is observed with psoralen crosslinking, ChEC, and Miller spreading. These observations are not incompatible if one considers that psoralen crosslinking indicates the presence of canonical nucleosomes, whereas ChIP analysis reveals presence of histone molecules. Histones might still be present on open rDNA copies, but a large body of evidence establishes that they are not arranged as canonical nucleosomes impermeable to psoralen. Alternative nucleosome structures have been described and occur specifically when DNA supercoiling is altered [96]. This observation agrees with older biochemical studies demonstrating that despite the absence of detectable beaded nucleosomes on active rRNA genes, the protein constituents of nucleosomes may still be present [8]. Moreover, by combining reagent accessibility analyses and electron microscopy of rDNA from Physarum polycephalum, the existence on active rRNA genes of an alternative nucleosome structure called the lexosome was suggested some time ago [97, 98]. The lexosome is an altered nucleosome specifically located on actively transcribed regions, which has properties that facilitate transcription. In a lexosome configuration, the histoneDNA interactions are different than those in intact nucleosomes and allow psoralen to access DNA. Therefore, even if this alternative structure was not confirmed when tested for in vitro transcription [99], the lexosome represents an attractive model that is consistent with the ChIP data, the psoralencrosslinking results, and the electron microscopy images produced in our studies as well as those of other research teams.
Similarly, the altered topology of actively transcribed rDNA might lead to other alternative histone configurations [96].

\section{Regulator of Pol I Elongation}

To date, most factors known to regulate Pol I elongation were characterized previously as Pol II elongation factors. Such dual functions make interpretation of phenotype difficult, since an indirect effect via Pol II is difficult to exclude. However, some Pol I elongation factors are now well characterized.

Spt5 copurifies with Pol I and has been shown to be associated with rDNA in vivo [100]. Spt5 is an evolutionarily conserved elongation factor with homologs found in eubacteria (NusG) and in archaea (RpoE) [101, 102]. Multisubunit RNA polymerases have the ability to stably interact with DNA through a structural feature called the DNA clamp. Prior to interacting with DNA, the DNA clamp must be in an open configuration and closed for processive elongation. Spt5/NusG can close the DNA clamp, making the polymerase able to carry out processive elongation [103]. Spt5 interacts with multiple Pol I subunits and Rrn3, and an Spt5 mutant (spt5-C292R) suppresses the growth defect of the rpa49D Pol I mutant [104]. Phenotypic analysis of rDNA transcription in the Spt5 mutant suggested that it positively and negatively regulates Pol I functions [105]. Other factors that interact with Spt5, such as Spt4, Paf1 and Spt6, have also been suggested to regulate Pol I. An spt4 deletion mutant led to decreased rDNA copy numbers, and an rRNA processing defect [100]. The Paf1, complex interacts physically with Spt5 [106] and is involved in stimulating Pol I elongation [107]. Spt6 is a histone chaperone that might also be a good candidate to regulate Pol I in vivo. In addition, the FACT complex stimulates Pol I activity in human cells [108]. FACT consists of Spt16 and Pob3, interacts with Spt5 in yeast [106], and is genetically linked to rpa49s [109]. Another Pol II transcription factor has been proposed to regulate Pol I: the Elongator, a six-subunit complex, conserved between yeast and mammals. In African trypanosomes, mutation or down-regulation of the Elp3b subunit of the complex results in increased synthesis of rRNA by Pol I [110].

The growing list of Pol II elongation factors that also regulate Pol I activity is interesting, but some mechanistic insights are still lacking. The elongation mechanisms of Pol II and Pol I are very different. Pol II carries regulators via interactions with CTD [111]. With few Pol II enzymes acting simultaneously on transcribed genes, the stoichiometric association of elongation factors with Pol II results in a density of about one elongation factor per gene. In contrast, a large number of Pol I enzymes can transcribe a single rRNA gene. Thus, it is difficult to imagine that Pol II elongation factors would be stochiometrically bound to each elongating Pol I. Therefore, it seems unlikely that Pol II and Pol I complexes use similar mechanisms of action or factor recruitment strategies. It remains to be understood how the same elongation factors can act in two very different transcription systems.

One factor can now be defined as a bona fide Pol I elongation factor, the nucleolin, called Nsr1 in budding yeast and 


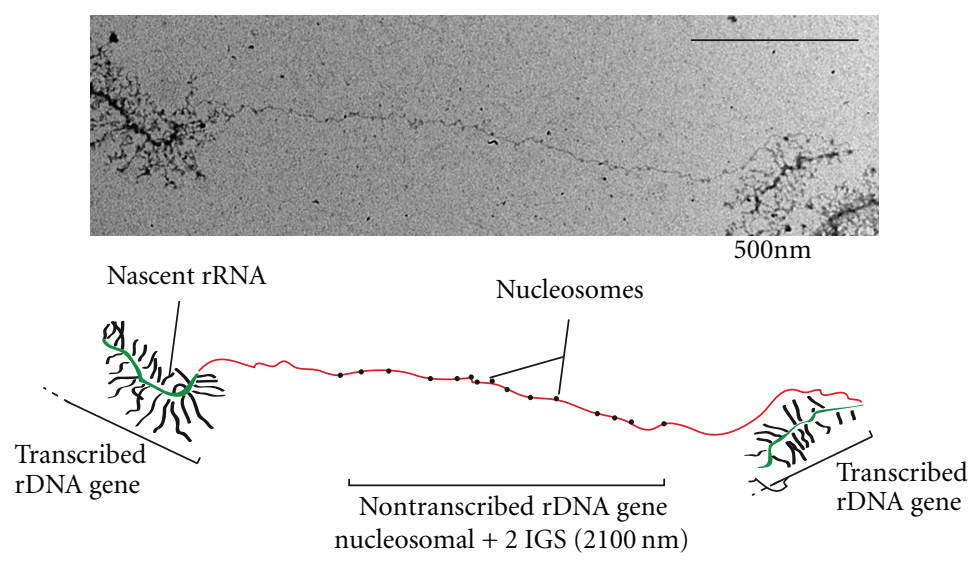

(a)
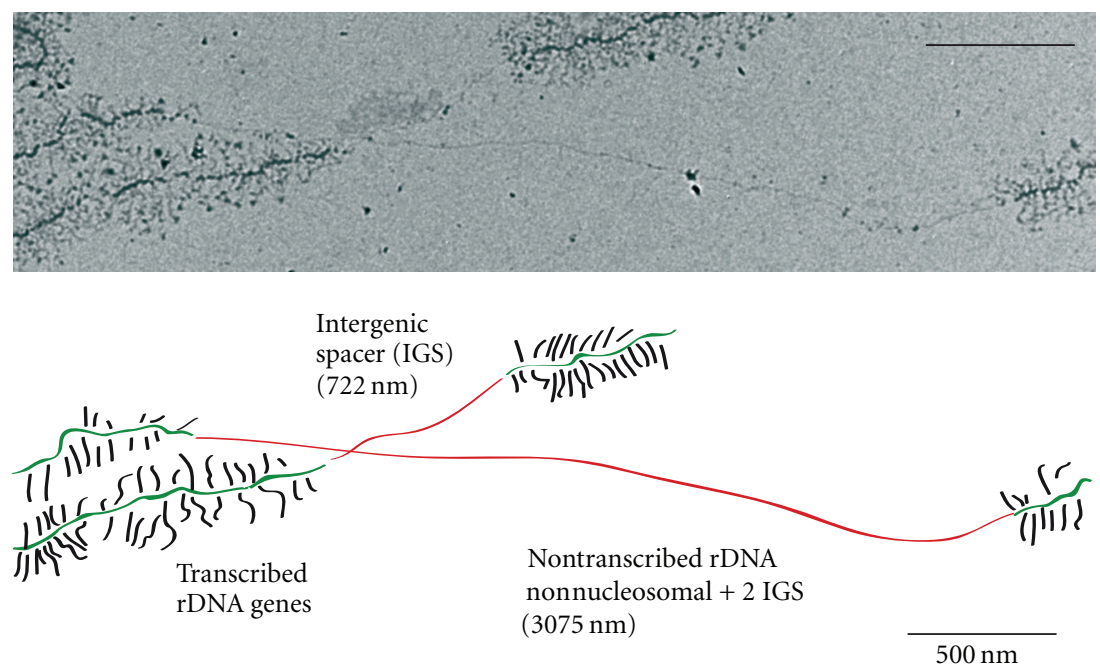

(b)

FIgURE 3: Miller spreading of nontranscribed rDNA. Single-gene analysis of nontranscribed rRNA genes reveals nucleosomal (a) and nonnucleosomal (b) organization. Chromatin spreading (upper panel) and a schematic representation of rDNA spreading (lower panel) are shown from strain NOY1071, bearing 25 rDNA copies. Transcribed rRNA genes (green) are identified by high Pol I density, and nascent rRNAs are shown in black. Non-transcribed regions are depicted in red. Intergenic spaces (IGSs) are short (600 nm) and can be easily distinguished from inactive rRNA genes. Inactive genes are flanked by two IGSs. Due to the DNA wrapping around nucleosomes, nontranscribed genes associated with nucleosomes appear shorter $(2,100 \mathrm{~nm}-(2 \times 600 \mathrm{~nm})=900 \mathrm{~nm})$ than genes devoid of nucleosomes $(3,100 \mathrm{~nm}-(2 \times 600)=1,900 \mathrm{~nm})$. With 15 nucleosomes detected and each wrapped around $146 \mathrm{bp}$, we observed a length reduction of approximately $1,000 \mathrm{~nm}$.

Gar2 in fission yeast. Following nucleolin's first identification as an abundant nucleolar protein, it has been implicated in numerous cellular processes $[112,113]$. Among them, it is clearly involved in ribosome biogenesis. Nucleolin is required for early rRNA-processing events [114] and for Pol I activity through a nucleosomal template [115]. Nucleolin has a histone chaperone activity and stimulates transcription by a mechanism reminiscent of the activity of the FACT complex [116]. Nucleolin is clearly an important factor to understand the interplay between rDNA chromatin, Pol I transcription, and cotranscriptional rRNA processing.

\section{Concluding Remarks}

In this paper, we tried to focus on the unanswered questions of rRNA production, rather than make an exhaustive review of the large body of work addressing regulation of this complex multistep process. We still know little about how cells adjust the production of each ribosomal constituent in time and space to allow cotranscriptional assembly of preribosomal particles. The answer to this question probably lies in the existence of highly redundant pathways, which are all designed to coregulate rRNA production by Pol I, ribosomal protein production, and the availability of the $\sim 200$ transacting factors required to assemble eukaryotic ribosomes. The discovery of redundant pathways has clearly resulted from the extensive study of budding yeast. Most regulatory pathways affecting rRNA production are not essential for cell growth. Out of 14 Pol I subunits, four are not required for cell growth. However, when double inactivation is performed, their functions can be revealed and studied [74]. We have no doubt that important progress remains to 
be made in understanding how Pol I is regulated. Because most of the complex interplay between rRNA production, assembly, cleavage, and folding occurs during elongation, we expect that most progress remaining to be made will uncover how rRNA elongation is coupled to rRNA assembly [81]. The central question of the exact structure and composition of open rDNA chromatin remains a major challenge. Pol I elongation is likely to be the most important step in controlling how nascent rRNA is folded and cleaved to yield pre-60S and pre-40S rRNA as they are being assembled into large preribosomes. We propose that elongation is the most regulatable step in rRNA production, making elongation the first target to regulate rRNA production.

\section{Acknowledgments}

The authors would like to thank all the members of the Gadal Lab for their contributions and critical evaluations of this paper. This work was supported by an ATIP-plus grant from CNRS, by the Agence Nationale de la Recherche (Nucleopol, Ribeuc and Jeune chercheur programme-ODynRib), and by Jeune Équipe from FRM. B. Albert is supported by a PhD fellowship from FRM. They thank Maxime Berthaud for preparing and quantifying Miller spreads obtained from mutant yeast cells. They also acknowledge Nacer Benmeradi and Stéphanie Balor for their help in EM image acquisition. This work also benefited from the assistance of the electron microscopy facility of the IFR 109 and from the imaging platform of Toulouse TRI.

\section{References}

[1] B. Albert, I. Leger-Silvestre, C. Normand et al., "RNA polymerase I-specific subunits promote polymerase clustering to enhance the rRNA gene transcription cycle," The Journal of Cell Biology, vol. 192, pp. 277-293, 2011.

[2] J. R. Warner, "The economics of ribosome biosynthesis in yeast," Trends in Biochemical Sciences, vol. 24, no. 11, pp. 437440, 1999.

[3] O. L. Miller and B. R. Beatty, "Visualization of nucleolar genes," Science, vol. 164, no. 3882, pp. 955-957, 1969.

[4] T. D. Petes, "Yeast ribosomal DNA genes are located on chromosome XII," Proceedings of the National Academy of Sciences of the United States of America, vol. 76, no. 1, pp. 410414, 1979.

[5] E. Schweizer and H. O. Halvorson, "On the regulation of ribosomal RNA synthesis in yeast," Experimental Cell Research, vol. 56, no. 2-3, pp. 239-244, 1969.

[6] T. Moss, F. Langlois, T. Gagnon-Kugler, and V. Stefanovsky, "A housekeeper with power of attorney: the rRNA genes in ribosome biogenesis," Cellular and Molecular Life Sciences, vol. 64, no. 1, pp. 29-49, 2007.

[7] S. L. French, Y. N. Osheim, F. Cioci, M. Nomura, and A. L. Beyer, "In exponentially growing Saccharomyces cerevisiae cells, rRNA synthesis is determined by the summed RNA polymerase I loading rate rather than by the number of active genes," Molecular and Cellular Biology, vol. 23, no. 5, pp. 1558-1568, 2003.

[8] A. Conconi, R. M. Widmer, T. Koller, and J. M. Sogo, "Two different chromatin structures coexist in ribosomal RNA genes throughout the cell cycle," Cell, vol. 57, no. 5, pp. 753$761,1989$.
[9] S. P. Bell, R. M. Learned, H. M. Jantzen, and R. Tjian, "Functional cooperativity between transcription factors UBF1 and SL1 mediates human ribosomal RNA synthesis," Science, vol. 241, no. 4870, pp. 1192-1197, 1988.

[10] J. C. B. M. Zomerdijk, H. Beckmann, L. Comai, and R. Tjian, "Assembly of transcriptionally active RNA polymerase I initiation factor SL1 from recombinant subunits," Science, vol. 266, no. 5193, pp. 2015-2018, 1994.

[11] J. J. Gorski, S. Pathak, K. Panov et al., "A novel TBP-associated factor of SL1 functions in RNA polymerase I transcription," EMBO Journal, vol. 26, no. 6, pp. 1560-1568, 2007.

[12] D. Eberhard, L. Tora, J. M. Egly, and I. Grummt, "A TBPcontaining multiprotein complex (TIF-IB) mediates transcription specificity of murine RNA polymerase I," Nucleic Acids Research, vol. 21, no. 18, pp. 4180-4186, 1993.

[13] C. A. Radebaugh, J. L. Matthews, G. K. Geiss et al., "TATA box-binding protein (TBP) is a constituent of the polymerase I- specific transcription initiation factor TIF-IB (SL1) bound to the rRNA promoter and shows differential sensitivity to TBP-directed reagents in polymerase I, II, and III transcription factors," Molecular and Cellular Biology, vol. 14, no. 1, pp. 597-605, 1994.

[14] J. Heix, J. C. B. M. Zomerdijk, A. Ravanpay, R. Tjian, and I. Grummt, "Cloning of murine RNA polymerase I-specific TAF factors: conserved interactions between the subunits of the species-specific transcription initiation factor TIFIB/SL1," Proceedings of the National Academy of Sciences of the United States of America, vol. 94, no. 5, pp. 1733-1738, 1997.

[15] V. Y. Stefanovsky, G. Pelletier, D. P. Bazett-Jones, C. CraneRobinson, and T. Moss, "DNA looping in the RNA polymerase I enhancesome is the result of non-cooperative inphase bending by two UBF molecules," Nucleic Acids Research, vol. 29, no. 15, pp. 3241-3247, 2001.

[16] D. P. Bazett-Jones, B. Leblanc, M. Herfort, and T. Moss, "Short-range DNA looping by the Xenopus HMG-box transcription factor, xUBF," Science, vol. 264, no. 5162, pp. 11341137, 1994.

[17] K. I. Panov, J. K. Friedrich, J. Russell, and J. C. B. M. Zomerdijk, "UBF activates RNA polymerase I transcription by stimulating promoter escape," EMBO Journal, vol. 25, no. 14, pp. 3310-3322, 2006.

[18] A. C. O’Sullivan, G. J. Sullivan, and B. McStay, "UBF binding in vivo is not restricted to regulatory sequences within the vertebrate ribosomal DNA repeat," Molecular and Cellular Biology, vol. 22, no. 2, pp. 657-668, 2002.

[19] V. Stefanovsky, F. Langlois, T. Gagnon-Kugler, L. I. Rothblum, and T. Moss, "Growth factor signaling regulates elongation of RNA polymerase I transcription in mammals via UBF phosphorylation and r-chromatin remodeling," Molecular Cell, vol. 21, no. 5, pp. 629-639, 2006.

[20] I. Grummt, "Wisely chosen paths-regulation of rRNA synthesis: delivered on 30 June 2010 at the 35th FEBS Congress in Gothenburg, Sweden," FEBS Journal, vol. 277, no. 22, pp. 4626-4639, 2010.

[21] D. A. Keys, L. Vu, J. S. Steffan et al., "RRN6 and RRN7 encode subunits of a multiprotein complex essential for the initiation of rDNA transcription by RNA polymerase I in Saccharomyces cerevisiae," Genes and Development, vol. 8, no. 19, pp. 2349-2362, 1994.

[22] D. Lalo, J. S. Steffan, J. A. Dodd, and M. Nomura, "RRN11 encodes the third subunit of the complex containing Rrn6p and Rrn7p that is essential for the initiation of rDNA transcription by yeast RNA polymerase I," Journal of Biological Chemistry, vol. 271, no. 35, pp. 21062-21067, 1996. 
[23] C. W. Lin, B. Moorefield, J. Payne, P. Aprikian, K. Mitomo, and R. H. Reeder, "A novel 66-kilodalton protein complexes with Rrn6, Rrn7, and TATA- binding protein to promote polymerase I transcription initiation in Saccharomyces cerevisiae," Molecular and Cellular Biology, vol. 16, no. 11, pp. 6436-6443, 1996.

[24] D. A. Keys, B. S. Lee, J. A. Dodd et al., "Multiprotein transcription factor UAF interacts with the upstream element of the yeast RNA polymerase I promoter and forms a stable preinitiation complex," Genes and Development, vol. 10, no. 7, pp. 887-903, 1996.

[25] M. Oakes, I. Siddiqi, L. Vu, J. Aris, and M. Nomura, “Transcription factor UAF, expansion and contraction of ribosomal DNA (rDNA) repeats, and RNA polymerase switch in transcription of yeast rDNA," Molecular and Cellular Biology, vol. 19, no. 12, pp. 8559-8569, 1999.

[26] L. Vu, I. Siddiqi, B. S. Lee, C. A. Josaitis, and M. Nomura, "RNA polymerase switch in transcription of yeast rDNA: Role of transcription factor UAF (upstream activation factor) in silencing rDNA transcription by RNA polymerase II," Proceedings of the National Academy of Sciences of the United States of America, vol. 96, no. 8, pp. 4390-4395, 1999.

[27] J. S. Steffan, D. A. Keys, J. A. Dodd, and M. Nomura, "The role of TBP in rDNA transcription by RNA polymerase I in Saccharomyces cerevisiae: TBP is required for upstream activation factor- dependent recruitment of core factor," Genes and Development, vol. 10, no. 20, pp. 2551-2563, 1996.

[28] O. Gadal, S. Labarre, C. Boschiero, and P. Thuriaux, "Hmo1, an HMG-box protein, belongs to the yeast ribosomal DNA transcription system," EMBO Journal, vol. 21, no. 20, pp. 5498-5507, 2002.

[29] A. H. Cavanaugh, A. Evans, and L. I. Rothblum, "Mammalian Rrn3 is required for the formation of a transcription competent preinitiation complex containing RNA polymerase I," Gene Expression, vol. 14, no. 3, pp. 131-147, 2007.

[30] R. T. Yamamoto, Y. Nogi, J. A. Dodd, and M. Nomura, "RRN3 gene of Saccharomyces cerevisiae encodes an essential RNA polymerase I transcription factor which interacts with the polymerase independently of DNA template," EMBO Journal, vol. 15, no. 15, pp. 3964-3973, 1996.

[31] P. Milkereit and H. Tschochner, "A specialized form of RNA polymerase I, essential for initiation and growth-dependent regulation of rRNA synthesis, is disrupted during transcription," EMBO Journal, vol. 17, no. 13, pp. 3692-3703, 1998.

[32] J. Bodem, G. Dobreva, U. Hoffmann-Rohrer et al., “TIF-IA, the factor mediating growth-dependent control of ribosomal RNA synthesis, is the mammalian homolog of yeast Rrn3p," EMBO Reports, vol. 1, no. 2, pp. 171-175, 2000.

[33] B. Moorefield, E. A. Greene, and R. H. Reeder, "RNA polymerase I transcription factor Rrn3 is functionally conserved between yeast and human," Proceedings of the National Academy of Sciences of the United States of America, vol. 97, no. 9, pp. 4724-4729, 2000.

[34] G. Peyroche, P. Milkereit, N. Bischler et al., "The recruitment of RNA polymerase I on rDNA is mediated by the interaction of the A43 subunit with Rrn3," EMBO Journal, vol. 19, no. 20, pp. 5473-5482, 2000.

[35] G. Miller, K. I. Panov, J. K. Friedrich, L. Trinkle-Mulcahy, A. I. Lamond, and J. C. B. M. Zomerdijk, "hRRN3 is essential in the SL1-mediated recruitment of RNA polymerase I to rRNA gene promoters," EMBO Journal, vol. 20, no. 6, pp. 1373 $1382,2001$.

[36] I. Hirschler-Laszkiewicz, A. H. Cavanaugh, A. Mirza et al., "Rrn3 becomes inactivated in the process of ribosomal DNA transcription," Journal of Biological Chemistry, vol. 278, no. 21, pp. 18953-18959, 2003.

[37] R. Loewith, E. Jacinto, S. Wullschleger et al., "Two TOR complexes, only one of which is rapamycin sensitive, have distinct roles in cell growth control," Molecular Cell, vol. 10, no. 3, pp. 457-468, 2002.

[38] J. A. Claypool, S. L. French, K. Johzuka et al., “Tor pathway regulates Rrn3p-dependent recruitment of yeast RNA polymerase I to the promoter but does not participate in alteration of the number of active genes," Molecular Biology of the Cell, vol. 15, no. 2, pp. 946-956, 2004.

[39] A. Laferté, E. Favry, A. Sentenac, M. Riva, C. Carles, and S. Chédin, "The transcriptional activity of RNA polymerase I is a key determinant for the level of all ribosome components," Genes and Development, vol. 20, no. 15, pp. 2030-2040, 2006.

[40] F. Beckouet, S. Labarre-Mariotte, B. Albert et al., "Two RNA polymerase I subunits control the binding and release of Rrn3 during transcription," Molecular and Cellular Biology, vol. 28 , no. 5, pp. 1596-1605, 2008.

[41] A. H. Cavanaugh, I. Hirschler-Laszkiewicz, Q. Hu et al., "Rrn3 phosphorylation is a regulatory checkpoint for ribosome biogenesis," Journal of Biological Chemistry, vol. 277, no. 30, pp. 27423-27432, 2002.

[42] J. Gerber, A. Reiter, R. Steinbauer et al., "Site specific phosphorylation of yeast RNA polymerase I," Nucleic Acids Research, vol. 36, no. 3, pp. 793-802, 2008.

[43] S. Rogers, R. Wells, and M. Rechsteiner, "Amino acid sequences common to rapidly degraded proteins: the PEST hypothesis," Science, vol. 234, no. 4774, pp. 364-368, 1986.

[44] A. Philippi, R. Steinbauer, A. Reiter et al., "TOR-dependent reduction in the expression level of Rrn3p lowers the activity of the yeast RNA Pol I machinery, but does not account for the strong inhibition of rRNA production," Nucleic Acids Research, vol. 38, no. 16, pp. 5315-5326, 2010.

[45] A. Reiter, R. Steinbauer, A. Philippi et al., "Reduction in ribosomal protein synthesis is sufficient to explain major effects on ribosome production after short-term TOR inactivation in Saccharomyces cerevisiae," Molecular and Cellular Biology, vol. 31, pp. 803-817, 2010.

[46] A. Huber, S. L. French, H. Tekotte et al., "Sch9 regulates ribosome biogenesis via Stb3, Dot6 and Tod6 and the histone deacetylase complex RPD3L," The EMBO Journal, vol. 30, no. 15, pp. 3052-3064, 2011.

[47] A. Huber, B. Bodenmiller, A. Uotila et al., "Characterization of the rapamycin-sensitive phosphoproteome reveals that Sch9 is a central coordinator of protein synthesis," Genes and Development, vol. 23, no. 16, pp. 1929-1943, 2009.

[48] C. Cadwell, H. J. Yoon, Y. Zebarjadian, and J. Carbon, "The yeast nucleolar protein Cbf5p is involved in rRNA biosynthesis and interacts genetically with the RNA polymerase I transcription factor RRN3," Molecular and Cellular Biology, vol. 17, no. 10, pp. 6175-6183, 1997.

[49] D. A. Rojas, S. Moreira-Ramos, S. Zock-Emmenthal et al., "Rrn7 protein, an RNA polymerase I transcription factor, is required for RNA polymerase II-dependent transcription directed by core promoters with a HomolD Box Sequence," The Journal of Biological Chemistry, vol. 286, pp. 2648026486, 2011.

[50] A. B. Berger, L. Decourty, G. Badis, U. Nehrbass, A. Jacquier, and O. Gadal, "Hmol is required for TOR-dependent regulation of ribosomal protein gene transcription," Molecular and Cellular Biology, vol. 27, no. 22, pp. 8015-8026, 2007.

[51] N. J. Proudfoot, A. Furger, and M. J. Dye, "Integrating mRNA processing with transcription," Cell, vol. 108 , no. 4, pp. 501$512,2002$. 
[52] S. Chanarat, M. Seizl, K. Strasser et al., "The Prp19 complex is a novel transcription elongation factor required for TREX occupancy at transcribed genes," Genes \& Development, vol. 25, pp. 1147-1158, 2011.

[53] S. Rodriguez-Navarro and E. Hurt, "Linking gene regulation to mRNA production and export," Current Opinion in Cell Biology, vol. 23, pp. 302-309, 2011.

[54] M. De La Mata, C. R. Alonso, S. Kadener et al., "A slow RNA polymerase II affects alternative splicing in vivo," Molecular Cell, vol. 12, no. 2, pp. 525-532, 2003.

[55] F. Dragon, J. E. G. Gallagher, P. A. Compagnone-Post et al., "A large nucleolar U3 ribonucleoprotein required for $18 \mathrm{~S}$ ribosomal RNA biogenesis," Nature, vol. 417, no. 6892, pp. 967-970, 2002.

[56] J. E. G. Gallagher, D. A. Dunbar, S. Granneman et al., "RNA polymerase I transcription and pre-rRNA processing are linked by specific SSU processome components," Genes and Development, vol. 18, no. 20, pp. 2507-2517, 2004.

[57] N. J. Krogan, W. T. Peng, G. Cagney et al., "High-definition macromolecular composition of yeast RNA-processing complexes," Molecular Cell, vol. 13, no. 2, pp. 225-239, 2004.

[58] M. Wery, S. Ruidant, S. Schillewaert, N. Leporé, and D. L. J. Lafontaine, "The nuclear poly(A) polymerase and Exosome cofactor Trf5 is recruited cotranscriptionally to nucleolar surveillance," RNA, vol. 15, no. 3, pp. 406-419, 2009.

[59] J. L. Prieto and B. McStay, "Recruitment of factors linking transcription and processing of pre-rRNA to NOR chromatin is UBF-dependent and occurs independent of transcription in human cells," Genes and Development, vol. 21, no. 16, pp. 2041-2054, 2007.

[60] S. Hofmann and O. L. Miller Jr., "Visualization of ribosomal ribonucleic acid synthesis in a ribonuclease III deficient strain of Escherichia coli," Journal of Bacteriology, vol. 132, no. 2, pp. 718-722, 1977.

[61] R. M. Grainger and N. Maizels, "Dictyostelium ribosomal RNA is processed during transcription," Cell, vol. 20, no. 3, pp. 619-623, 1980.

[62] Y. N. Osheim, S. L. French, K. M. Keck et al., "Pre-18S ribosomal RNA is structurally compacted into the SSU processome prior to being cleaved from nascent transcripts in Saccharomyces cerevisiae," Molecular Cell, vol. 16, no. 6, pp. 943-954, 2004.

[63] M. Koš and D. Tollervey, "Yeast Pre-rRNA Processing and Modification Occur Cotranscriptionally," Molecular Cell, vol. 37, no. 6, pp. 809-820, 2010.

[64] J. Pérez-Fernández, A. Román, J. De Las Rivas, X. R. Bustelo, and M. Dosil, "The $90 \mathrm{~S}$ preribosome is a multimodular structure that is assembled through a hierarchical mechanism," Molecular and Cellular Biology, vol. 27, no. 15, pp. 5414-5429, 2007.

[65] A. Segerstolpe, P. Lundkvist, Y. N. Osheim, A. L. Beyer, and L. Wieslander, "Mrd1p binds to pre-rRNA early during transcription independent of U3 snoRNA and is required for compaction of the pre-rRNA into small subunit processomes," Nucleic Acids Research, vol. 36, no. 13, pp. 4364-4380, 2008.

[66] Y. Nogi, R. Yano, and M. Nomura, "Synthesis of large rRNAs by RNA polymerase II in mutants of Saccharomyces cerevisiae defective in RNA polymerase I," Proceedings of the National Academy of Sciences of the United States of America, vol. 88, no. 9, pp. 3962-3966, 1991.

[67] M. Oakes, Y. Nogi, M. W. Clark, and M. Nomura, "Structural alterations of the nucleolus in mutants of Saccharomyces cerevisiae defective in RNA polymerase I," Molecular and Cellular Biology, vol. 13, no. 4, pp. 2441-2455, 1993.
[68] S. Trumtel, I. Léger-Silvestre, P. E. Gleizes, F. Teulières, and N. Gas, "Assembly and functional organization of the nucleolus: ultrastructural analysis of Saccharomyces cerevisiae mutants," Molecular Biology of the Cell, vol. 11, no. 6, pp. 21752189, 2000.

[69] S. Fath, P. Milkereit, A. V. Podtelejnikov et al., "Association of yeast RNA polymerase I with a nucleolar substructure active in rRNA synthesis and processing," Journal of Cell Biology, vol. 149, no. 3, pp. 575-590, 2000.

[70] D. A. Schneider, A. Michel, M. L. Sikes et al., "Transcription elongation by RNA polymerase I is linked to efficient rRNA processing and ribosome assembly," Molecular Cell, vol. 26, no. 2, pp. 217-229, 2007.

[71] C. D. Kuhn, S. R. Geiger, S. Baumli et al., "Functional architecture of RNA polymerase I," Cell, vol. 131, no. 7, pp. 1260-1272, 2007.

[72] K. I. Panov, T. B. Panova, O. Gadal et al., "RNA polymerase I-specific subunit CAST/hPAF49 has a role in the activation of transcription by upstream binding factor," Molecular and Cellular Biology, vol. 26, no. 14, pp. 5436-5448, 2006.

[73] L. F. Liu and J. C. Wang, "Supercoiling of the DNA template during transcription," Proceedings of the National Academy of Sciences of the United States of America, vol. 84, no. 20, pp. 7024-7027, 1987.

[74] O. Gadal, S. Mariotte-Labarre, S. Chedin et al., "A34.5, a nonessential component of yeast RNA polymerase I, cooperates with subunit A14 and DNA topoisomerase I to produce a functional rRNA synthesis machine," Molecular and Cellular Biology, vol. 17, no. 4, pp. 1787-1795, 1997.

[75] S. Gangloff, J. P. McDonald, C. Bendixen, L. Arthur, and R. Rothstein, "The yeast type I topoisomerase top3 interacts with Sgs1, a DNA helicase homolog: a potential eukaryotic reverse gyrase," Molecular and Cellular Biology, vol. 14, no. 12, pp. 8391-8398, 1994.

[76] M. F. Christman, F. S. Dietrich, N. A. Levin, B. U. Sadoff, and G. R. Fink, "The rRNA-encoding DNA array has an altered structure in topoisomerase I mutants of Saccharomyces cerevisiae," Proceedings of the National Academy of Sciences of the United States of America, vol. 90, no. 16, pp. 7637-7641, 1993.

[77] R. P. Perry and D. E. Kelley, "Inhibition of RNA synthesis by actinomycin D: characteristic dose-response of different RNA species," Journal of Cellular Physiology, vol. 76, no. 2, pp. 127-139, 1970.

[78] B. Sollner-Webb and J. Tower, "Transcription of cloned eukaryotic ribosomal RNA genes," Annual Review of Biochemistry, vol. 55, pp. 801-830, 1986.

[79] K. V. Hadjiolova, A. A. Hadjiolov, and J. P. Bachellerie, "Actinomycin D stimulates the transcription of rRNA minigenes transfected into mouse cells. Implications for the in vivo hypersensitivity of rRNA gene transcription," European Journal of Biochemistry, vol. 228, no. 3, pp. 605-615, 1995.

[80] D. K. Trask and M. T. Muller, "Stabilization of type I topoisomerase-DNA covalent complexes by actinomycin D," Proceedings of the National Academy of Sciences of the United States of America, vol. 85, no. 5, pp. 1417-1421, 1988.

[81] A. El Hage, S. L. French, A. L. Beyer, and D. Tollervey, "Loss of Topoisomerase I leads to R-loop-mediated transcriptional blocks during ribosomal RNA synthesis," Genes and Development, vol. 24, no. 14, pp. 1546-1558, 2010.

[82] S. L. French, M. L. Sikes, R. D. Hontz et al., "Distinguishing the roles of Topoisomerases I and II in relief of transcriptioninduced torsional stress in yeast rRNA genes," Molecular and Cellular Biology, vol. 31, pp. 482-494, 2010. 
[83] S. J. Brill, S. DiNardo, K. Voelkel-Meiman, and R. Sternglanz, "Need for DNA topoisomerase activity as a swivel for DNA replication for transcription of ribosomal RNA," Nature, vol. 326, no. 6111, pp. 414-416, 1987.

[84] C. Lavelle, "DNA torsional stress propagates through chromatin fiber and participates in transcriptional regulation," Nature Structural and Molecular Biology, vol. 15, no. 2, pp. 123-125, 2008.

[85] D. A. Koster, V. Croquette, C. Dekker, S. Shuman, and N. H. Dekker, "Friction and torque govern the relaxation of DNA supercoils by eukaryotic topoisomerase IB," Nature, vol. 434, no. 7033, pp. 671-674, 2005.

[86] J. Salceda, X. Fernández, and J. Roca, “Topoisomerase II, not topoisomerase $\mathrm{I}$, is the proficient relaxase of nucleosomal DNA," EMBO Journal, vol. 25, no. 11, pp. 2575-2583, 2006.

[87] B. U. Sadoff, S. Heath-Pagliuso, I. B. Castano, Y. Zhu, F. S. Kieff, and M. F. Christman, "Isolation of mutants of Saccharomyces cerevisiae requiring DNA topoisomerase I," Genetics, vol. 141, no. 2, pp. 465-479, 1995.

[88] R. Dammann, R. Lucchini, T. Koller, and J. M. Sogo, "Chromatin structures and transcription of rDNA in yeast Saccharomyces cerevisiae," Nucleic Acids Research, vol. 21, no. 10, pp. 2331-2338, 1993.

[89] M. Schmid, T. Durussel, and U. K. Laemmli, "ChIC and ChEC: genomic mapping of chromatin proteins," Molecular Cell, vol. 16, no. 1, pp. 147-157, 2004.

[90] K. Merz, M. Hondele, H. Goetze, K. Gmelch, U. Stoeckl, and J. Griesenbeck, "Actively transcribed rRNA genes in S. cerevisiae are organized in a specialized chromatin associated with the high-mobility group protein $\mathrm{Hmol}$ and are largely devoid of histone molecules," Genes and Development, vol. 22, no. 9, pp. 1190-1204, 2008.

[91] A. Chindamporn, S. I. Iwaguch, Y. Nakagawa, M. Homma, and K. Tanaka, "Clonal size-variation of rDNA cluster region on chromosome XII of Saccharomyces cerevisiae," Journal of General Microbiology, vol. 139, no. 7, pp. 1409-1415, 1993.

[92] T. Kobayashi, D. J. Heck, M. Nomura, and T. Horiuchi, "Expansion and contraction of ribosomal DNA repeats in Saccharomyces cerevisiae: requirement of replication fork blocking (Fob1) protein and the role of RNA polymerase I," Genes and Development, vol. 12, no. 24, pp. 3821-3830, 1998.

[93] R. Lucchini and J. M. Sogo, "Replication of transcriptionally active chromatin," Nature, vol. 374, no. 6519, pp. 276-280, 1995.

[94] M. Wittner, S. Hamperl, U. Stockl et al., "Establishment and maintenance of alternative chromatin States at a multicopy gene locus," Cell, vol. 145, pp. 543-554, 2011.

[95] H. S. Jones, J. Kawauchi, P. Braglia, C. M. Alen, N. A. Kent, and N. J. Proudfoot, "RNA polymerase I in yeast transcribes dynamic nucleosomal rDNA," Nature Structural and Molecular Biology, vol. 14, no. 2, pp. 123-130, 2007.

[96] C. Lavelle and A. Prunell, "Chromatin polymorphism and the nucleosome superfamily: a genealogy," Cell Cycle, vol. 6, no. 17, pp. 2113-2119, 2007.

[97] C. P. Prior, C. R. Cantor, E. M. Johnson, and V. G. Allfrey, "Incorporation of exogenous pyrene-labeled histone into Physarum chromatin: a system for studying changes in nucleosomes assembled in vivo," Cell, vol. 20, no. 3, pp. 597 $608,1980$.

[98] H. S. Judelson and V. M. Vogt, "Accessibility of ribosomal genes to trimethyl psoralen in nuclei of Physarum polycephalum," Molecular and Cellular Biology, vol. 2, no. 3, pp. 211220, 1982.
[99] R. U. Protacio and J. Widom, "Nucleosome transcription studied in a real-time synchronous system: test of the lexosome model and direct measurement of effects due to histone octamer," Journal of Molecular Biology, vol. 256, no. 3, pp. 458-472, 1996.

[100] D. A. Schneider, S. L. French, Y. N. Osheim et al., "RNA polymerase II elongation factors Spt $4 p$ and Spt5p play roles in transcription elongation by RNA polymerase I and rRNA processing," Proceedings of the National Academy of Sciences of the United States of America, vol. 103, no. 34, pp. 1270712712, 2006.

[101] F. W. Martinez-Rucobo, S. Sainsbury, A. C. Cheung, and P. Cramer, "Architecture of the RNA polymerase-Spt4/5 complex and basis of universal transcription processivity," The EMBO Journal, vol. 30, pp. 1302-1310, 2011.

[102] B. J. Klein, D. Bose, K. J. Baker et al., "RNA polymerase and transcription elongation factor Spt $4 / 5$ complex structure," Proceedings of the National Academy of Sciences of the United States of America, vol. 108, pp. 546-550, 2011.

[103] G. A. Hartzog, T. Wada, H. Handa, and F. Winston, "Evidence that Spt4, Spt5, and Spt6 control transcription elongation by RNA polymerase II in Saccharomyces cerevisiae," Genes and Development, vol. 12, no. 3, pp. 357-369, 1998.

[104] O. V. Viktorovskaya, F. D. Appling, and D. A. Schneider, "Yeast transcription elongation factor Spt5 associates with RNA polymerase I and RNA polymerase II directly," The Journal of Biological Chemistry, vol. 286, pp. 18825-18833, 2011.

[105] S. J. Anderson, M. L. Sikes, Y. Zhang et al., "The transcription elongation factor spt5 influences transcription by RNA polymerase I positively and negatively," The Journal of Biological Chemistry, vol. 286, pp. 18816-18824, 2011.

[106] S. L. Squazzo, P. J. Costa, D. L. Lindstrom et al., "The Paf1 complex physically and functionally associates with transcription elongation factors in vivo," EMBO Journal, vol. 21, no. 7, pp. 1764-1774, 2002.

[107] Y. Zhang, M. L. Sikes, A. L. Beyer, and D. A. Schneider, "The Pafl complex is required for efficient transcription elongation by RNA polymerase I," Proceedings of the National Academy of Sciences of the United States of America, vol. 106, no. 7, pp. 2153-2158, 2009.

[108] J. L. Birch, B. C. M. Tan, K. I. Panov et al., "FACT facilitates chromatin transcription by RNA polymerases i and III," EMBO Journal, vol. 28, no. 7, pp. 854-865, 2009.

[109] A. P. Davierwala, J. Haynes, Z. Li et al., "The synthetic genetic interaction spectrum of essential genes," Nature Genetics, vol. 37, no. 10, pp. 1147-1152, 2005.

[110] S. Alsford and D. Horn, "Elongator protein 3b negatively regulates ribosomal DNA transcription in african trypanosomes," Molecular and Cellular Biology, vol. 31, pp. 18221832,2011

[111] A. Mayer, M. Lidschreiber, M. Siebert, K. Leike, J. Söding, and P. Cramer, "Uniform transitions of the general RNA polymerase II transcription complex," Nature Structural and Molecular Biology, vol. 17, no. 10, pp. 1272-1278, 2010.

[112] L. R. Orrick, M. O. J. Olson, and H. Busch, "Comparison of nucleolar proteins of normal rat liver and Novikoff hepatoma ascites cells by two dimensional polyacrylamide gel electrophoresis," Proceedings of the National Academy of Sciences of the United States of America, vol. 70, no. 5, pp. 1316-1320, 1973.

[113] F. Mongelard and P. Bouvet, "Nucleolin: a multiFACeTed protein," Trends in Cell Biology, vol. 17, no. 2, pp. 80-86, 2007. 
[114] H. Ginisty, F. Amalric, and P. Bouvet, "Nucleolin functions in the first step of ribosomal RNA processing," EMBO Journal, vol. 17, no. 5, pp. 1476-1486, 1998.

[115] B. Rickards, S. J. Flint, M. D. Cole, and G. LeRoy, "Nucleolin is required for RNA polymerase I transcription in vivo," Molecular and Cellular Biology, vol. 27, no. 3, pp. 937-948, 2007.

[116] D. Angelov, V. A. Bondarenko, S. Almagro et al., "Nucleolin is a histone chaperone with FACT-like activity and assists remodeling of nucleosomes," EMBO Journal, vol. 25, no. 8, pp. 1669-1679, 2006. 

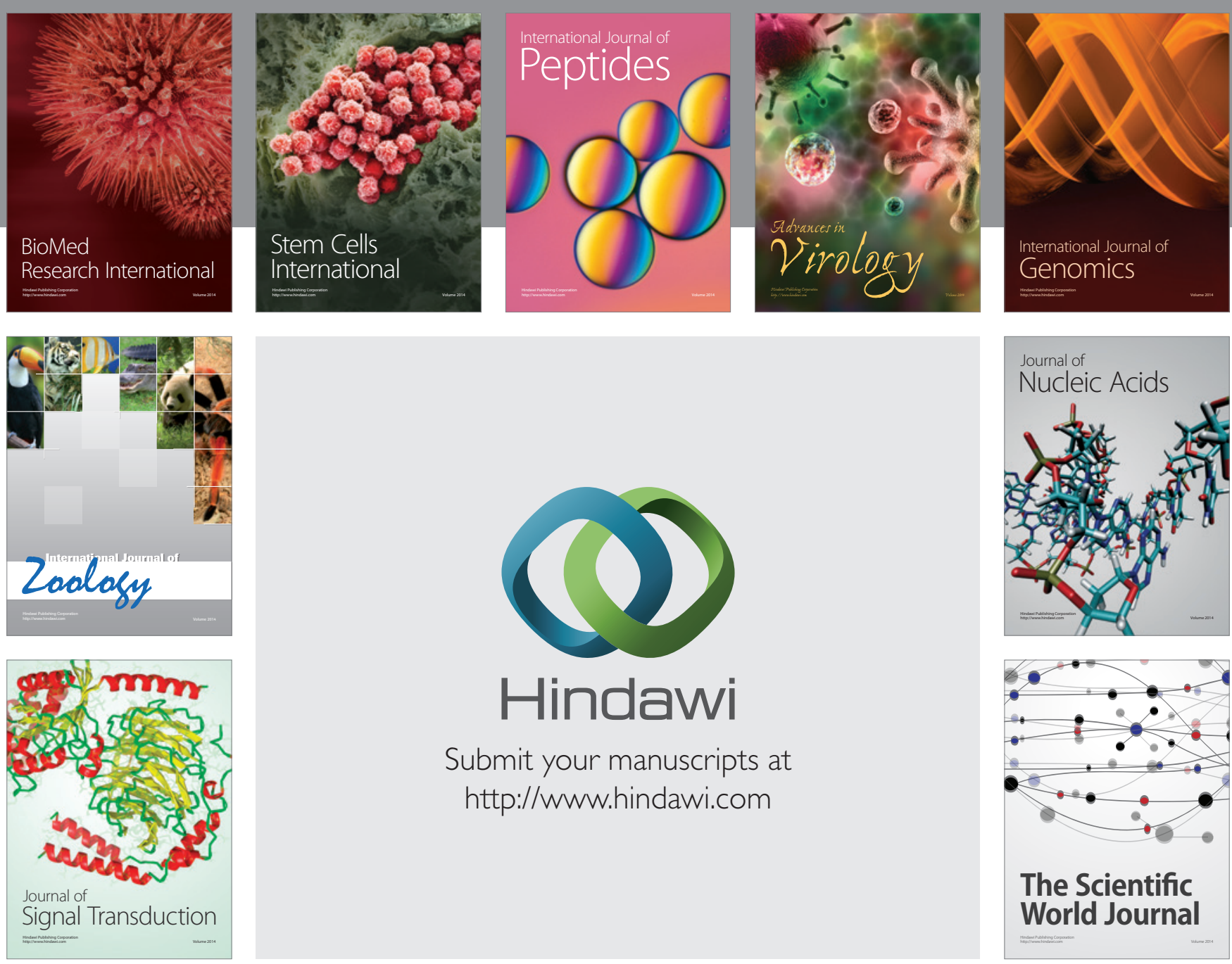

Submit your manuscripts at

http://www.hindawi.com
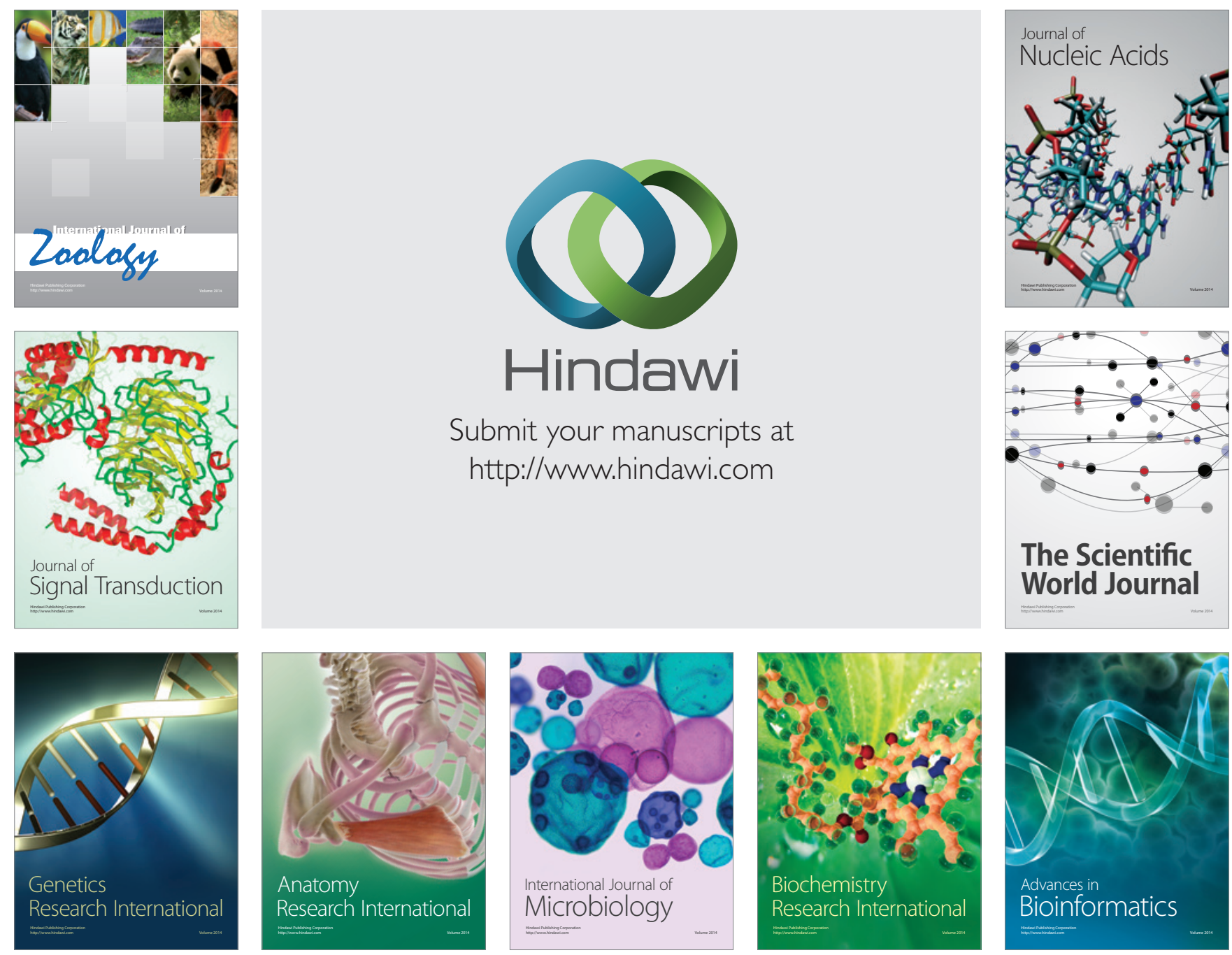

The Scientific World Journal
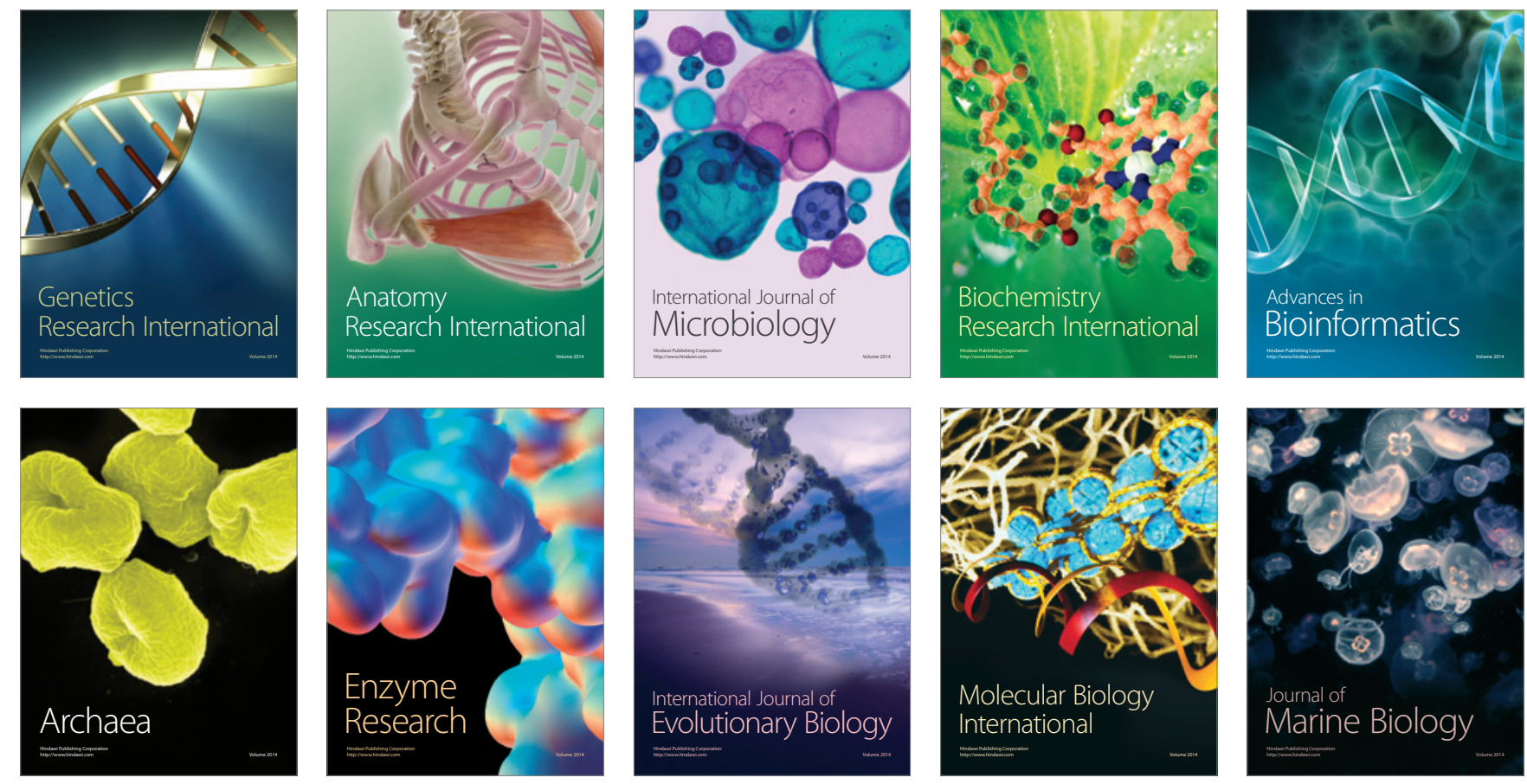